\title{
89. Electron Microscopy on the Blue Back of a Clupeoid Fish, Harengula zunasi*
}

\author{
By Siro Kawaguti and Yoshihisa Kamishima \\ Department of Biology, Faculty of Science, \\ Okayama University \\ (Comm. by Yô K. OKada, M.J.A., April 12, 1966)
}

In many kinds of fish such as scombroids and clupeoids, the dorsal side is blue or greenish blue with various dark patterns differing from species to species, but the ventral side or belly appeares silvery white. These fish are surface swimmers, and we may consider that both the blue dorsal and the white ventral side are effective in helping the fish to conceal themselves in their surrounding water. The water is blue when it is seen from above and glittering white seen from below.

Since the work of Ballowitz ${ }^{1}$ it has been generally accepted that such blue color on the dorsal side is caused by the presence of iridophores cushioned on melanophores, whereas, the ventral white is composed of a thick accumulation of guanophores without melanophores. ${ }^{2)}$ Detailed structures of these iridophores and guanophores are important to understand their roles in producing these color phenomena. However, they have remained concealed beyond the limit of the light microscope.

In our previous papers on the green color of the Japanese wrasse $^{3)}$ and on the blue spots of the Japanese porgy, ${ }^{4)}$ we have demonstrated iridophores having lamellar structures. Recently it has been proved that each of these lamellae has thin guanine platelets of various thicknesses and extensions. ${ }^{5}$ This paper deals with electron micrographs of the iridophores in the blue dorsal skin of a clupeoid fish. These pictures show clearly the interrelations between lamellar bodies and guanine platelets in the iridophore.

The material used in this experiment was a clupeoid fish, Harengula zunasi BLEEKER, which was collected near the Tamano Marine Laboratory. The fish is blue on the dorsal side with a faint yellow line on each lateral side and glittering white on the ventral side. The dorsal blue color, however, changes according to environmental conditions. It turns pale grey in a dark place, but it is blue under bright light or when the fish is killed, owing to the expansion and contraction of melanophores respectively.

\footnotetext{
*) Contribution from the Tamano Marine Laboratory, No. 151.
} 
Methods for treatment of materials and for observations were the same as described in previous papers on iridophores. ${ }^{3,4)}$ Special care has been taken to demonstrate the thin guanine platelets which were found in each interspace or cistern between lamellar bodies. $^{4)}$

Chromatophores are usually found below the thick connective tissue of the skin. Fig. 1 shows a part of a chromatophore layer under the thick connective tissue. The layer is composed of three elements, xanthophores, iridophores, and melanophores. Xanthophores are very thin and have only small vesicular bodies showing quite different features from those of the xanthophores of the treefrog. ${ }^{6)}$ They sometimes disappear, leaving a loose connective tissue and some cellular extensions between the thick connective tissue and the iridophores.

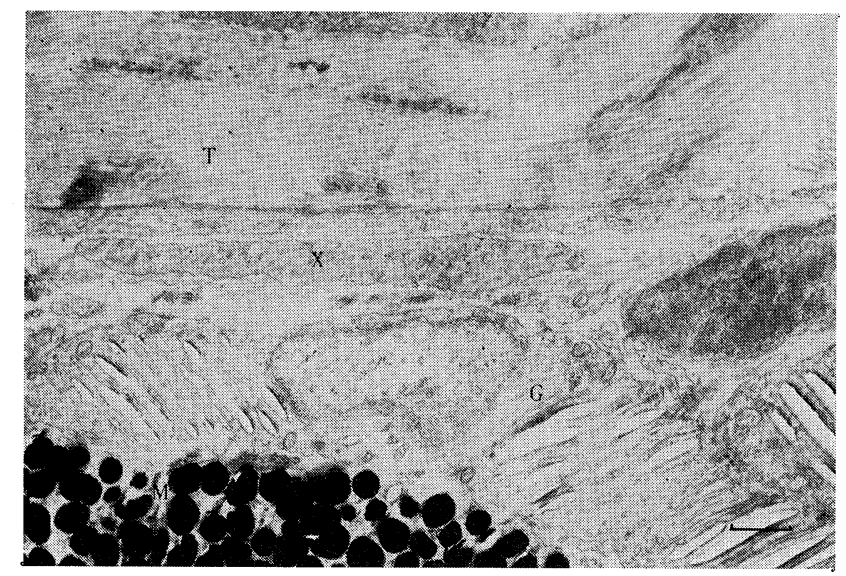

Fig. 1. A chromatophore layer of the dorsal blue skin of a clupeoid fish. $T$, thick connective tissue; $X$, xanthophore; $G$, iridophore; $M$, melanophore. $\times 5800$

The iridophore is very large, measuring $10 \mu$ in breadth and $5 \mu$ in thickness. It appears as a cup form filled with a large nucleus as is shown in Fig. 2. The upper side of the nucleus is covered with only a thin film of the cytoplasm. The other sides are surrounded by several groups of lamellar bodies. These lamellar bodies are various in length and number in one group, ranging from 6 to 12 , but they are almost uniform in their thickness which measures from 700 to $1200 \mathrm{~A}$. Each lamellar body shows rather wavy outlines and is separated from each neighboring one by a rather clear interspace. The structures of these lamellar bodies are similar to those of the iridophores of the Japanese wrasse, the Japanese porgy and also of the green tree-frog. The clear interspace is frequently 


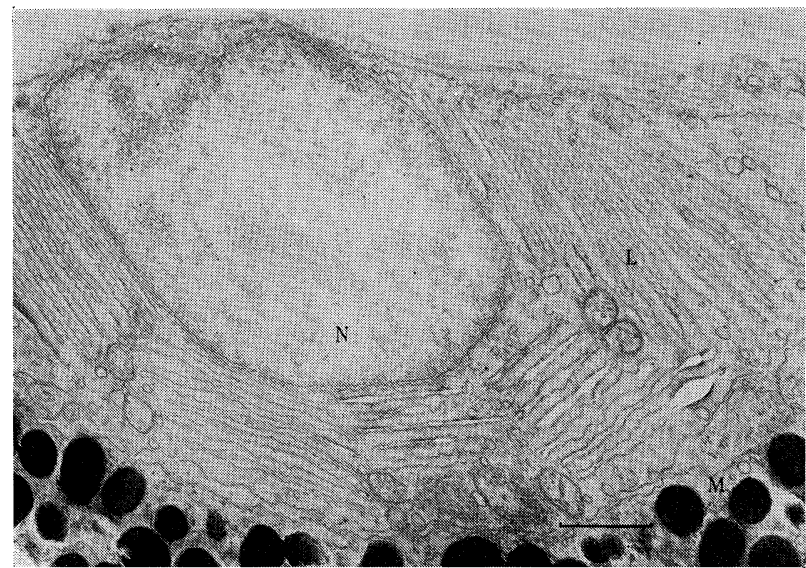

Fig. 2. An enlarged view of an iridophore showing a large nucleus and surrounding groups of lamellar bodies. $N$, nucleus; $L$, lamellar bodies. $\times 9000$

observed as an empty space, but in some preparations there is a thin dark line running down the middle.

Fig. 3 shows an enlarged view of such dark lines in the interspaces. These dark lines run straight from one end to the opposite end of the interspace, though frequently interrupted in the middle portion. They appear in such straight lines in every section; that is, they must be sections of thin platelets. These platelets measure

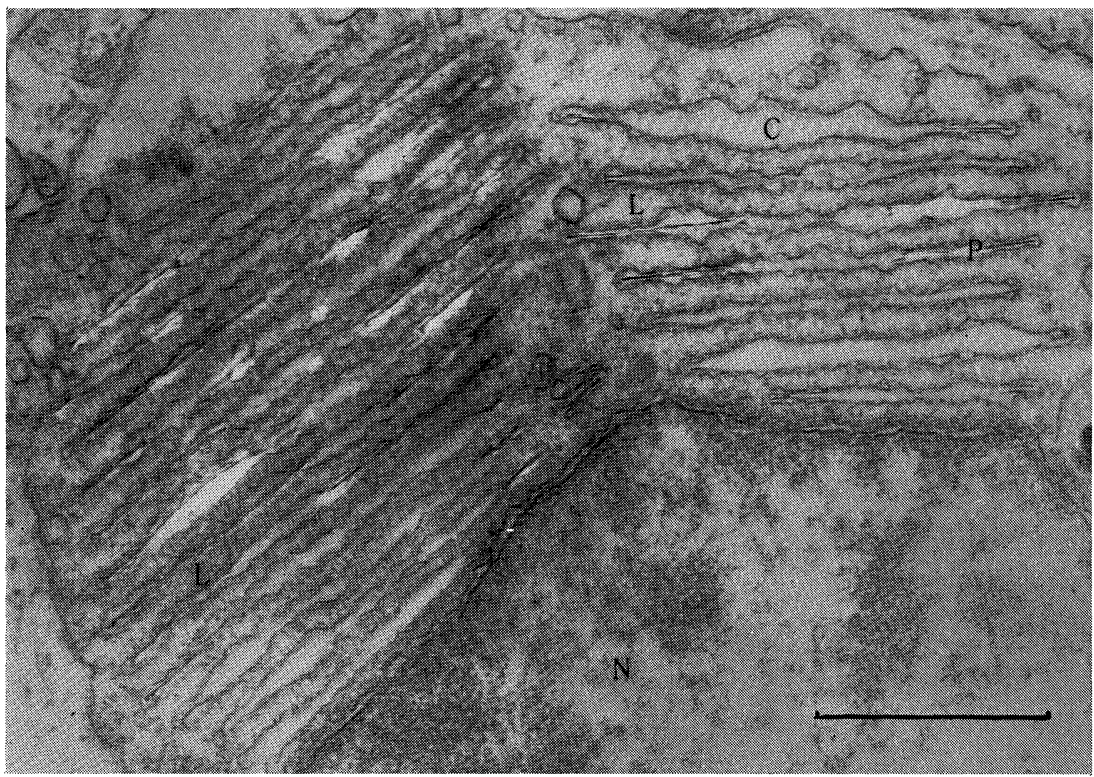

Fig. 3. An enlarged picture of two groups of lamellar bodies showing guanine platelets within the interspaces. Platelets appear as thin dark lines which are frequently broken. $C$, interspaces or cisterns; $P$, guanine platelets. $\times 24000$ 
about $1 \mu \times 3 \mu$ in breadth and $60 \mathrm{~A}$ in thickness. From these pictures we may suppose that these platelets are the first indications of guanine crystals which have been secreted into these interspaces and have crystalized there. When these platelets become thicker with the addition of further layers of crystals, they will turn into brittle substances which are easily destroyed and disappear from the electron microscopic preparations.

It was fortunate to find rather wide interspaces which may hold guanine platelets in the iridophores of this clupeoid fish during electron microscopic preparations. In the blue wrasse, such interspaces are very narrow as has been reported in a previous paper ${ }^{3)}$ and are not big enough to retain such thin platelets during preparation. When the guanine platelets become thicker, they disappear in both fish. Guanine platelets are found parallel to each other within one group, at distances of about $1700 \mathrm{~A}$. In some groups they show somewhat wider distances, which may partly be caused by the obliqueness of the cut plane.

Very thick guanine platelets of about $1000 \mathrm{~A}$ in thickness are found in the iridophores even of the clupeoid fish as has also been reported in those of the blue spots of the Japanese porgy. In the white belly of the clupeoid fish, guanophores are small and are distributed under the scale in a thick layer, without a background of melanophores. Under the electron microscope these guanophores have a full content of small guanine platelets about $1 \mu$ square and $0.1 \mu$ in thickness. These features of the guanophores in the white belly are essentially the same as those of the iridophores in the blue back except for the smaller dimensions and greater thickness of the guanine platelets. That is, the iridophores in the blue back are modified guanophores. This also holds true of the iridophores of the blue wrasse and the Japanese porgy.

The green colors of the wrasse may mainly be caused by the presence of xanthophores which contain pterin as an yellow filter for blue light reflected through the iridophores. Xanthophores in the blue wrasse are found so closely associated with iridophores that in the previous paper $^{3)}$ the author failed to locate the pterin correctly. The structure of guanophores will deserve a further description in a separate paper.

The melanophores are seated at the bottom of the chromatophore layer. They have many dark melanine granules of various dimensions. Around the iridophores there are some thin protoplasmic extensions, some of which are extensions of the melanophores and contain melanine granules when the skin has turned pale. 


\section{References}

1) Ballowitz, E. (1914): Z. Wiss. Zool., 110, 1-35.

2) Fox, H. M., and G. Vevers (1960): The Nature of Animal Colours. Sidgwick and Jackson, London.

3) Kawaguti, S. (1965): Proc. Japan Acad., 41, 610-613.

4) Kawaguti, S., and Y. Kamishima (1964): Biol. Jour. Okayama Univ., 10. 7581.

5) - (1966): Ibid., 12, 57-60.

6) Kawaguti, S., Y. Kamishima, and K. Sato (1965): Ibid., 11, 97-109. 